\title{
THE ROMAN BONDAGE NUMBER OF A DIGRAPH
}

\author{
N. DEHGARDI, D. MEIERLING, S. M. SHEIKHOLESLAMI AND L. VOLKMANN
}

\begin{abstract}
Let $D=(V, A)$ be a finite and simple digraph. A Roman dominating function on $D$ is a labeling $f: V(D) \rightarrow\{0,1,2\}$ such that every vertex with label 0 has an in-neighbor with label 2. The weight of an $\operatorname{RDF} f$ is the value $\omega(f)=\sum_{v \in V} f(\nu)$. The minimum weight of a Roman dominating function on a digraph $D$ is called the Roman domination number, denoted by $\gamma_{R}(D)$. The Roman bondage number $b_{R}(D)$ of a digraph $D$ with maximum out-degree at least two is the minimum cardinality of all sets $A^{\prime} \subseteq A$ for which $\gamma_{R}\left(D-A^{\prime}\right)>$ $\gamma_{R}(D)$. In this paper, we initiate the study of the Roman bondage number of a digraph. We determine the Roman bondage number in several classes of digraphs and give some sharp bounds.
\end{abstract}

\section{Introduction}

Let $D$ be a finite simple digraph with vertex set $V(D)=V$ and arc set $A(D)=A$. A digraph without directed cycles of length 2 is an oriented graph. The order $n=n(D)$ of a digraph $D$ is the number of its vertices. We write $\operatorname{deg}_{D}^{+}(\nu)=\operatorname{deg}^{+}(v)$ for the outdegree of a vertex $v$ and $\operatorname{deg}_{D}^{-}(\nu)=\operatorname{deg}^{-}(\nu)$ for its indegree. The minimum and maximum indegree and minimum and maximum outdegree of $D$ are denoted by $\delta^{-}=\delta^{-}(D), \Delta^{-}=\Delta^{-}(D), \delta^{+}=\delta^{+}(D)$ and $\Delta^{+}=$ $\Delta^{+}(D)$, respectively. If $(u, v)$ is an arc of $D$, then we also write $u \rightarrow v$, and we say that $v$ is an out-neighbor of $u$ and $u$ is an in-neighbor of $v$. For a vertex $v$ of a digraph $D$, we denote the set of in-neighbors and out-neighbors of $v$ by $N^{-}(\nu)=N_{D}^{-}(v)$ and $N^{+}(v)=N_{D}^{+}(v)$, respectively. If $X \subseteq V(D)$, then $D[X]$ is the subdigraph induced by $X$. If $X \subseteq V(D)$ and $v \in V(D)$, then $A(X, v)$ is the set of arcs from $X$ to $v$. Note that for any digraph $D$ with $m$ arcs,

$$
\sum_{u \in V(D)} \operatorname{deg}^{-}(u)=\sum_{u \in V(D)} \operatorname{deg}^{+}(u)=m
$$

The underlying graph $G[D]$ of a digraph $D$ is that graph obtained by replacing each arc $u v$ by an edge $u v$. Note that $G[D]$ has two parallel edges $u v$ when $D$ contains the $\operatorname{arcs}(u, v)$ and

Received December 3, 2015, accepted June 23, 2016.

2010 Mathematics Subject Classification. 05C69.

Key words and phrases. Roman dominating function, Roman domination number, Roman bondage number, digraph.

Corresponding author: S. M. Sheikholeslami. 
$(\nu, u)$. A digraph $D$ is called connected, if the underlying graph $G[D]$ is connected. Consult $[9,20]$ for the notation and terminology which are not defined here. For a real-valued function $f: V(D) \longrightarrow \mathbb{R}$, the weight of $f$ is $w(f)=\sum_{\nu \in V} f(\nu)$. For $S \subseteq V$, we define $f(S)=\sum_{\nu \in S} f(\nu)$, so $w(f)=f(V)$.

A subset $S$ of vertices of $D$ is a dominating set if $N^{+}[S]=V$. The domination number $\gamma(D)$ is the minimum cardinality of a dominating set of $D$. The bondage number, denoted by $b(D)$, of $D$ is the minimum number of arcs whose removal from $D$ results in a digraph with larger domination number. An arc set $B$ for which $\gamma(D-B)>\gamma(D)$ is called a bondage set. A $b(D)$-set is a bondage set of $D$ of size $b(D)$. If $B$ is a $b(D)$-set, then obviously

$$
\gamma(D-B)=\gamma(D)+1
$$

Recently, Carlson and Develin [3], Shan and Kang [16], and Huang and Xu [10, 12] studied the bondage number for digraphs, independently. A thorough study of bondage number appears in [19].

A Roman dominating function (RDF) on a digraph $D=(V, A)$ is a function $f: V \longrightarrow\{0,1,2\}$ satisfying the condition that every vertex $v$ for which $f(v)=0$ has a in-neighbor $u$ for which $f(u)=2$. The weight of an $\operatorname{RDF} f$ is the value $\omega(f)=\sum_{\nu \in V} f(\nu)$. The minimum weight of a Roman dominating function on a digraph $D$ is called the Roman domination number, denoted by $\gamma_{R}(D)$. A $\gamma_{R}(D)$-function is a Roman dominating function of $D$ with weight $\gamma_{R}(D)$. Roman domination for digraphs is investigated in $[6,17]$. A Roman dominating function $f: V \longrightarrow$ $\{0,1,2\}$ can be represented by the ordered partition $\left(V_{0}, V_{1}, V_{2}\right)$ (or $\left(V_{0}^{f}, V_{1}^{f}, V_{2}^{f}\right)$ to refer to $f$ ) of $V$, where $V_{i}=\{v \in V \mid f(v)=i\}$. In this representation, its weight is $\omega(f)=\left|V_{1}\right|+2\left|V_{2}\right|$. It is clear that

$$
\gamma(D) \leq \gamma_{R}(D) \leq 2 \gamma(D)
$$

The Roman dominating number for undirected graphs was introduced by Steward [18] and ReVelle and Rosing [15] and has been studied by several authors [4, 5, 8].

The Roman bondage number $b_{R}(D)$ of a digraph $D$ is the cardinality of a smallest set of $\operatorname{arcs} A^{\prime} \subseteq A(D)$ for which $\gamma_{R}\left(D-A^{\prime}\right)>\gamma_{R}(D)$.

The definition of the Roman bondage number for undirected graphs was given by Jafari Rad and Volkmann [13] and has been studied by several authors [2, 14, 7].

Our purpose in this paper is to initiate the study of the Roman bondage number in digraphs. We first present some general upper bounds for Roman bondage number and then we determine the Roman bondage number of some classes of digraphs.

By Proposition $\mathrm{B}$, we note that if $D$ is a digraph with $\Delta^{+}(D) \leq 1$, then $\gamma_{R}(D)=n$ implying that for any subset $A^{\prime} \subseteq A(D), \gamma_{R}\left(D-A^{\prime}\right)=\gamma_{R}(D)$. Therefore the Roman bondage number is only defined for digraphs with maximum out-degree at least two. 
We make use of the following results in this paper.

\section{Proposition A ([17]).}

(1) For a digraph $D$ of order $n \geq 2, \gamma_{R}(D)=2$ if and only if $\Delta^{+}(D)=n-1$ or $n=2$ and $A(D)=\varnothing$.

(2) For a digraph $D$ oforder $n \geq 3, \gamma_{R}(D)=3$ if and only if $\Delta^{+}(D)=n-2$ or $n=3$ and $\Delta^{+}(D) \leq 1$.

(3) For a digraph $D$ of order $n \geq 4, \gamma_{R}(D)=4$ if and only if $\Delta^{+}(D)=n-3$ or $\Delta^{+}(D) \leq n-3$ and there are two vertices $u, v \in V(D)$ such that $N_{D}^{+}[u] \cup N_{D}^{+}[v]=V(D)$ or $n=4$ and $\Delta^{+}(D) \leq 1$.

Proposition B ([17]). Let D be a digraph of order $n$. Then $\gamma_{R}(D)<n$ if and only if $\Delta^{+}(D) \geq 2$.

For every graph $G$, the expression $\operatorname{deg}_{a}(G)=\sum_{\nu \in V(G)} \operatorname{deg}(\nu) /|V(G)|$ is called the average degree of $G$.

Proposition $\mathrm{C}([1])$. For any digraph $D$ with $\delta^{-}(D) \geq 1$, there exists a pair of vertices, say $u$ and $v$, that are either adjacent or at distance two in $G[D]$ with a common in-neighbor in $D$, with the property that

$$
\operatorname{deg}_{G[D]}(u)+\operatorname{deg}_{G[D]}(\nu) \leq 2 \operatorname{deg}_{a}(G[D])
$$

Proposition D ([11]). For any vertex transitive digraph $D$ of order $n, b(D) \geq\left\lceil\frac{n}{\gamma(D)}\right\rceil$.

Observation 1. Let $D$ be a digraph of order $n$ with $\gamma_{R}(D)<n$. Assume that $H$ is a spanning subdigraph of $D$ with $\gamma_{R}(H)=\gamma_{R}(D)$. If $K=A(D)-A(H)$, then $b_{R}(H) \leq b_{R}(D) \leq b_{R}(H)+|K|$.

Proof. Let $F \subseteq A(D)$ be a set such that $\gamma_{R}(D-F)>\gamma_{R}(D)$ and $|F|=b_{R}(D)$. It follows that $\gamma_{R}(H-F) \geq \gamma_{R}(D-F)>\gamma_{R}(D)=\gamma_{R}(H)$ and hence $b_{R}(H) \leq|F|=b_{R}(D)$.

Now let $F^{\prime} \subseteq A(H)$ such that $\gamma_{R}\left(H-F^{\prime}\right)>\gamma_{R}(H)$ and $\left|F^{\prime}\right|=b_{R}(H)$. We deduce that $\gamma_{R}(D-$ $\left.\left(K \cup F^{\prime}\right)\right)=\gamma_{R}\left(H-F^{\prime}\right)>\gamma_{R}(H)=\gamma_{R}(D)$ and thus $b_{R}(D) \leq b_{R}(H)+|K|$.

Observation 2. If a digraph $D$ has a vertex $v$ such that every $\gamma_{R}(D)$-function assigns 2 to $v$, then $b_{R}(D) \leq \operatorname{deg}^{+}(v) \leq \Delta^{+}$.

Proof. Let $A_{v}^{+}$be the set of arcs in $D$ with tail $v$. Assume that $f$ is a $\gamma_{R}\left(D-A_{v}^{+}\right)$-function. Clearly $f$ is a Roman dominating function of $D$. Since $N_{D-A_{\nu}^{+}}^{+}(\nu)=\varnothing$, we deduce that $f(\nu) \neq 2$ and hence $f$ is not a $\gamma_{R}(D)$-function. It follows that $\gamma_{R}\left(D-A_{v}^{+}\right)>\gamma_{R}(D)$, and the proof is complete.

\section{Bounds on the Roman bondage number}

In this section we establish bounds on the Roman bondage number of a digraph. 
Theorem 3. If $D$ is a digraph, and $x y z$ a path of length 2 in $G[D]$ such that $(y, x),(y, z) \in A(D)$, then

$$
b_{R}(D) \leq \operatorname{deg}_{G[D]}(x)+\operatorname{deg}_{G[D]}(y)+\operatorname{deg}_{G[D]}(z)-3-\left|N^{-}(x) \cap N^{-}(y)\right| .
$$

Moreover, if $x$ and $z$ are adjacent in $G[D]$, then

$$
b_{R}(D) \leq \operatorname{deg}_{G[D]}(x)+\operatorname{deg}_{G[D]}(y)+\operatorname{deg}_{G[D]}(z)-4-\left|N^{-}(x) \cap N^{-}(y)\right| .
$$

Proof. Let $D^{\prime}$ be the digraph obtained from $D$ by removing the arcs incident with $x, y$ and $z$ with the exception of $(y, z)$ and all arcs going from $N^{-}(x) \cap N^{-}(y)$ to $y$. In $D^{\prime}$, the vertex $x$ is isolated, $z$ is a vertex with indegree 1 and outdegree 0 or 1 , and $y$ is an in-neighbor of $z$ and all in-neighbors of $y$ in $D^{\prime}$, if any, lie in $N^{-}(x)$. Let $f=\left(V_{0}, V_{1}, V_{2}\right)$ be a $\gamma_{R}\left(D^{\prime}\right)$-function. Then $f(x)=1$. Consider three cases.

Case 1. $f(z)=0$.

Then $f(y)=2$ and therefore $\left(V_{0} \cup\{x\}, V_{1}-\{x\}, V_{2}\right)$ is an RDF on $D$ of weight less than $\omega(f)$, and (4) as well as (5) are proved.

Case 2. $f(z)=2$.

Then $f(y)=0$ and the function $g: V(D) \rightarrow\{0,1,2\}$ defined by $g(x)=0, g(y)=2, g(z)=0$ and $g(v)=f(v)$ otherwise, is clearly an RDF on $D$ of weight less than $\omega(f)$, and hence (4) as well as (5) hold.

Case 3. $f(z)=1$.

If $f(y)=1$, then $\left(V_{0} \cup\{x, z\}, V_{1}-\{x, y, z\}, V_{2} \cup\{y\}\right)$ is an RDF on $D$ of weight less than $f$, and (4) as well as (5) hold. However, if $f(y)=0$, then there exists a vertex $w \in N^{-}(x) \cap N^{-}(y)$ such that $f(w)=2$. Since $w$ is an in-neighbor of $x$ in $\mathrm{D},\left(V_{0} \cup\{x\}, V_{1}-\{x\}, V_{2}\right)$ is an RDF on $D$ of weight less than $\omega(f)$, and the proof is complete.

By applying Theorem 3, we obtain the following result that gives a lower bound on the number of arcs in a digraph with given Roman bondage number.

Theorem 4. Let $D$ be a digraph of order $n$ with $\delta^{-}(D) \geq 1, \delta^{+}(D) \geq 2$ and Roman bondage number $b_{R}(D)$. If $\operatorname{deg}_{a}(G[D])$ is the average degree of the underlying graph of $D$, then

$$
b_{R}(D) \leq 2 \operatorname{deg}_{a}(G[D])+\Delta(G[D])-3 \text { and }|A(D)| \geq(n / 4)\left(b_{R}(D)-\Delta(G[D])+3\right) .
$$

Proof. Let $D$ be a digraph satisfying the hypothesis. By Proposition $\mathrm{C}$, we know that there is at least one pair of vertices, say $u$ and $v$, that are either adjacent or at distance 2 from each other with a common in-neighbor, and with the property that $\operatorname{deg}_{G[D]}(u)+\operatorname{deg}_{G[D]}(v) \leq$ $2 \operatorname{deg}_{a}(G[D])$. If $u$ and $v$ are at distance two in $G[D]$ with a common in-neighbor $w$ in $D$, then $u w v$ is a path in $G[D]$ such that $(w, u),(w, v) \in A(D)$. If $u$ and $v$ are adjacent and $(v, u) \in A(D)$, 
then it follows from $\delta^{+}(D) \geq 2$ that $v$ has an out-neighbor other than $u$, say $w$. In each case, there is a path $x y z$ in $G[D]$ such that $(y, x),(y, z) \in A(D)$. Using Theorem 3, we obtain

$$
b_{R}(D) \leq \operatorname{deg}_{G[D]}(x)+\operatorname{deg}_{G[D]}(y)+\operatorname{deg}_{G[D]}(z)-3 \leq 2 \operatorname{deg}_{a}(G[D])+\Delta(G[D])-3 .
$$

Since $2|E(G[D])|=n \operatorname{deg}_{a}(G[D])$, we have $4|E(G[D])|=2 n \operatorname{deg}_{a}(G[D]) \geq n\left(b_{R}(D)-\Delta(G[D])+\right.$ 3). Hence

$$
|A(D)|=|E(G[D])| \geq(n / 4)\left(b_{R}(D)-\Delta(G[D])+3\right) .
$$

Theorem 5. If $D$ is a digraph, and $x y z$ a path of length 2 in $G[D]$ such that $(y, x),(y, z) \in A(D)$, then

$$
b_{R}(D) \leq \operatorname{deg}_{G[D]}(x)+\operatorname{deg}_{D}^{-}(y)+\operatorname{deg}_{G[D]}(z)-\left|N^{-}(x) \cap N^{-}(y) \cap N^{-}(z)\right| .
$$

Proof. Let $S$ be the set of all arcs incident to $x, z$ and all arcs terminating at $y$ with the exception of all arcs going from $N^{-}(x) \cap N^{-}(z)$ to $y$. By construction, $|S|=\operatorname{deg}_{G[D]}(x)+\operatorname{deg}^{-}(y)+$ $\operatorname{deg}_{G[D]}(z)-\left|N^{-}(x) \cap N^{-}(y) \cap N^{-}(z)\right|$. Let $D^{\prime}$ be the digraph obtained from $D$ by removing the $\operatorname{arcs}$ of $S$. In $D^{\prime}$, the vertices $x, z$ are isolated, and all in-neighbors of $y$ in $D^{\prime}$, if any, lie in $N^{-}(x) \cap N^{-}(z)$. Let $f=\left(V_{0}, V_{1}, V_{2}\right)$ be a $\gamma_{R}\left(D^{\prime}\right)$-function. Then $f(x)=f(z)=1$.

If $f(y)=2$, then $\left(V_{0} \cup\{x, z\}, V_{1}-\{x, z\}, V_{2}\right)$ is an RDF on $D$ of weight less than $f$. If $f(y)=1$, then $\left(V_{0} \cup\{x, z\}, V_{1}-\{x, y, z\}, V_{2} \cup\{y\}\right)$ is an RDF on $D$ of weight less than $f$. However, if $f(y)=0$, then there exists a vertex $w \in N^{-}(x) \cap N^{-}(y) \cap N^{-}(z)$ such that $f(w)=2$. Since $w$ is an inneighbor of $x$ and $z$ in $D,\left(V_{0} \cup\{x, z\}, V_{1}-\{x, z\}, V_{2}\right)$ is an RDF on $D$ of weight less than $f$, and the proof is complete.

Assume that $\delta^{+}(D) \geq 2$, and let $y \in V(D)$ be an arbitrary vertex. Then there exist two different vertices $x, z \in N^{+}(y)$. Thus $G[D]$ contains a path $x y z$ such that $(y, x),(y, z) \in A(D)$ for each vertex $y$ in $D$. Applying Theorem 5 for a vertex $y \in V(D)$ with $\operatorname{deg}^{-}(y)=\delta^{-}(D)$, we obtain the next bound immediately.

Corollary 6. If $D$ is a digraph with $\delta^{+}(D) \geq 2$, then $b_{R}(D) \leq 2 \Delta(G[D])+\delta^{-}(D)$.

Since $\delta^{-}(D) \leq \frac{1}{2} \Delta(G[D])$, this in turn yields the next corollary.

Corollary 7. If $D$ is a digraph with $\delta^{+}(D) \geq 2$, then $b_{R}(D) \leq \frac{5}{2} \Delta(G[D])$.

The next result presents an upper bound on the Roman bondage number that involves the maximum degree. This bound also indicates a relationship between the Roman bondage number and the Roman domination number.

Theorem 8. Let $D$ be a digraph of order $n \geq 4$ with $\delta^{+}(D) \geq 2$ and Roman domination number $\gamma_{R}(D) \geq 3$. Then

$$
b_{R}(D) \leq\left(\gamma_{R}(D)-2\right) \Delta(G[D])+1 \text {. }
$$


Proof. By Corollary 6, the result is immediate for $\gamma_{R}(D) \geq 5$. Henceforth, we assume that $\gamma_{R}(D) \leq 4$. First assume that $\gamma_{R}(D)=3$. Let $u$ be a vertex in $D$, and let $A_{u}$ denote the set of arcs incident with $u$. If $\gamma_{R}\left(D-A_{u}\right)>\gamma_{R}(D)$, then $b_{R}(D) \leq\left|A_{u}\right|=\operatorname{deg}_{G[D]}(u)$ and hence $b_{R}(D) \leq$ $\Delta(G[D])$. Thus we may assume that $\gamma_{R}\left(D-A_{u}\right)=\gamma_{R}(D)$ or equivalently $\gamma_{R}(D-u)=\gamma_{R}(D)-1=$ 2 for every vertex $u \in V(D)$. Let $V(D)=\left\{v_{1}, v_{2}, \ldots, v_{n}\right\}$. Since $\gamma_{R}\left(D-v_{i}\right)=2$, there is a vertex $\hat{v}_{i}$ such that every vertex of $D-\left\{v_{i}, \hat{v}_{i}\right\}$ is an out-neighbor of $\hat{v}_{i}$. Hence $\operatorname{deg}^{+}\left(\hat{v}_{i}\right) \geq n-2$ for each $i$. On the other hand, $\gamma_{R}(D)=3$ implies that $\operatorname{deg}^{+}\left(\hat{v}_{i}\right)=n-2$ for each $i$. Define $\hat{g}: V(D) \rightarrow V(D)$ by $\hat{g}\left(\nu_{i}\right)=\hat{v}_{i}$ for $i=1, \ldots, n$. It follows from $\gamma_{R}(D)=3$ that $\hat{v}_{i} \neq \hat{v}_{j}$ if $i \neq j$. Hence, the function $\hat{g}: V(D) \rightarrow V(D)$ is one to one and so $V(D)=\left\{\hat{v}_{1}, \hat{v}_{2}, \ldots, \hat{v}_{n}\right\}$. This implies that $\operatorname{deg}^{+}\left(v_{i}\right)=n-2$ for each $i$. Let $A_{\nu_{1}}$ denote the set of arcs incident with $\nu_{1}$ and let $a$ be an arc with tail $\hat{\nu}_{1}$. Then $\gamma_{R}\left(D-A_{\nu_{1}}-a\right)>3=\gamma_{R}(D)$ and hence $b_{R}(D) \leq \operatorname{deg}_{G[D]}\left(\nu_{1}\right)+1 \leq \Delta(G[D])+1$.

Now assume that $\gamma_{R}(D)=4$. Suppose, to the contrary, that $b_{R}(D)>2 \Delta(G[D])+1$. Then for any vertex $u$ of $D$, we have $\gamma_{R}(D-u)=\gamma_{R}(D)-1=3$, since $\operatorname{deg}_{G[D]}(u)<b_{R}(D)$. Let $u \in V(D)$ and let $A_{u}$ denote the set of arcs incident with $u$. Assume that $H=D-A_{u}$. Then clearly $\gamma_{R}(H)=\gamma_{R}(D)$ and $b_{R}(H)=b_{R}(D-u)$. By Observation 1 we have $b_{R}(D) \leq b_{R}(H)+\left|A_{u}\right|=$ $b_{R}(D-u)+\operatorname{deg}_{G[D]}(u)$. Since $\gamma_{R}(D-u)=3$, we have

$$
\begin{aligned}
b_{R}(D) & \leq b_{R}(D-u)+\operatorname{deg}_{G[D]}(u) \\
& \leq \Delta(G[D-u])+1+\operatorname{deg}_{G[D]}(u) \\
& \leq \Delta(G[D])+1+\Delta(G[D]) \\
& =2 \Delta(G[D])+1 .
\end{aligned}
$$

This contradiction completes the proof.

For a digraph $D$, a subset $S$ of $V(D)$ and $x \in S$, the private out-neighborhood of $x$ with respect to $S$ is the set $P N(x, S)=N^{+}[x]-N^{+}[S-\{x\}]$. If $f=\left(V_{0}^{f}, V_{1}^{f}, V_{2}^{f}\right)$ is a $\gamma_{R}(D)$-function, then every vertex $x$ of $V_{2}^{f}$ has at least two private out-neighbors with respect to $V_{2}^{f}$, one of them being possibly $x$ and the other ones in $V_{0}^{f}$. If a vertex $x$ of $V_{2}^{f}$ has exactly one private out-neighbor $y$ in $V_{0}^{f}$, we can also put $x$ and $y$ in $V_{1}^{f}$. To avoid this ambiguity, we choose in this case to put $x$ and $y$ in $V_{1}^{f}$ and we call $\operatorname{good} \gamma_{R}(D)$-function, a $\gamma_{R}(D)$-function such that $\left|V_{2}^{f}\right|$ is minimum. Then every vertex of $V_{2}^{f}$ has at least two private out-neighbors with respect to $V_{2}^{f}$, obviously all in $V_{0}^{f}$.

We now introduce a parameter to bound $b_{R}(D)$. Let $a$ be an $\operatorname{arc}$ of $D$, and let $f=\left(V_{0}, V_{1}, V_{2}\right)$ be a $\gamma_{R}(D)$-function. We say that the arc a supports $f$ if $a \in A\left(V_{2}, V_{0}\right)=\left\{(u, v) \mid u \in V_{2}, v \in V_{0}\right\}$. Denote by $s(D)$ the minimum size of a set of arcs such that each $\gamma_{R}(D)$-function is supported by at least one such arc. Three $\operatorname{arcs} a, b, c$ support all $\gamma_{R}(D)$-functions in Figure 1.

Theorem 9. For any digraph $D, b_{R}(D) \geq s(D)$ with equality if for any $\gamma_{R}(D)$-function $f=$ $\left(V_{0}, V_{1}, V_{2}\right)$, each $u \in V_{0}$ is a private out-neighbor of some vertex in $V_{2}$. 


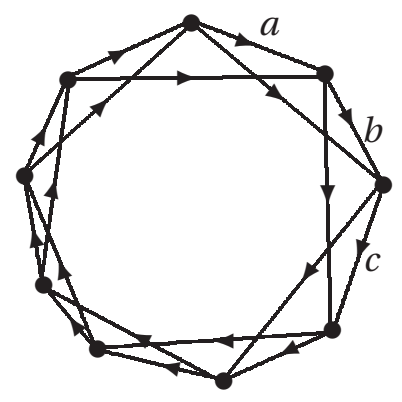

Figure 1: A digraph $D$ with $b_{R}(D)=s(D)=3$

Proof. Assume $A^{\prime} \subseteq A(D)$ with $\left|A^{\prime}\right|<s(D)$. Then there exists a $\gamma_{R}(D)$-functions $f=\left(V_{0}, V_{1}, V_{2}\right)$ such that $f$ is not supported by any arc in $A^{\prime}$. We show by contradiction that $f$ is still a $\gamma_{R}(D-$ $A^{\prime}$ )-function.

Suppose to the contrary that there exists a vertex $u \in V_{0}$ such that $u \notin N^{+}\left(V_{2}\right)$ in $D-$ $A^{\prime}$. Since $f$ is a $\gamma_{R}(D)$-function, there exists a vertex $v \in V_{2}$ such that $(\nu, u) \in A(D)$. Hence $(\nu, u) \in A(D)$ supports $f$, which implies that $(\nu, u) \notin A^{\prime}$. It follows that $u \in N_{D-A^{\prime}}^{+}(\nu)$ which is a contradiction. Thus $\gamma_{R}(D)=\gamma_{R}\left(D-A^{\prime}\right)$ for any set $A^{\prime} \subseteq A(D)$ with $\left|A^{\prime}\right|<s(D)$, and so $b_{R}(D) \geq s(D)$.

Now let $D$ be a digraph and let for any $\gamma_{R}(D)$-function $f=\left(V_{0}, V_{1}, V_{2}\right)$, each $u \in V_{0}$ be a private out-neighbor of some vertex in $V_{2}$. Assume that $f=\left(V_{0}, V_{1}, V_{2}\right)$ is an arbitrary $\gamma_{R}(D)$ function. Assume that $A^{\prime}$ is a set of $s(D)$ arcs such that each $\gamma_{R}(D)$-function is supported by at least one such arc. We show that $f$ is not an RDF of $D-A^{\prime}$. Since $A^{\prime}$ supports $f$, there exists an $\operatorname{arc}(\nu, u) \in A^{\prime}$ such that $v \in V_{2}, u \in V_{0}$ and $u$ is a private out-neighbor of $v$. By the assumption, $u \notin N_{D-A^{\prime}}^{+}\left(V_{2}\right)$. Thus $f$ is not an RDF of $D-A^{\prime}$. It follows that $\gamma_{R}\left(D-A^{\prime}\right)>\gamma_{R}(D)$ and so $b_{R}(D)=s(D)$. This completes the proof.

Proposition 10. For every digraph $D$ with $\gamma_{R}(D)=2 \gamma(D)$,

$$
b_{\mathrm{R}}(D) \geq b(D) \text {. }
$$

Proof. Let $B$ be a $b_{R}(D)$ - set. Then by (3) we have

$$
2 \gamma(D-B) \geq \gamma_{\mathrm{R}}(D-B)>\gamma_{\mathrm{R}}(D)=2 \gamma(D)
$$

Thus $\gamma(D-B)>\gamma(D)$ and hence $b_{\mathrm{R}}(D) \geq b(D)$.

We close this section with presenting a lower bound on the Roman bondage number of vertex-transitive digraphs. 
Theorem 11. Let $D$ be a vertex-transitive digraph of order $n$. Then

$$
b_{R}(D) \geq\left\lceil\frac{2 n}{\gamma_{R}(D)}\right\rceil \text {. }
$$

Proof. Let $V(D)=\left\{v_{1}, v_{2}, \ldots, v_{n}\right\}$ and let $\mathscr{D}_{i}$ be the family of all good $\gamma_{R}(D)$-functions $f$ that assign 2 to $v_{i}$. By definition, we observe that if $f=\left(V_{0}^{f}, V_{1}^{f}, V_{2}^{f}\right) \in \mathscr{D}_{i}$ and $g=\left(V_{0}^{g}, V_{1}^{g}, V_{2}^{g}\right) \in$ $\mathscr{D}_{j}$, then $r=\left|V_{2}^{f}\right|=\left|V_{2}^{g}\right|$.

First we show that $\left|\mathscr{D}_{i}\right|=\left|\mathscr{D}_{j}\right|$ for any $i$ and $j$. Since $D$ is vertex-transitive, there exists an automorphism $\theta$ of $D$ such that $\theta\left(v_{i}\right)=v_{j}$. It is not hard to see that for any good $\gamma_{R}(D)$ function $f \in \mathscr{D}_{i}$, we have $f \theta^{-1} \in \mathscr{D}_{j}$. Define the function $\psi: \mathscr{D}_{i} \rightarrow \mathscr{D}_{j}$ by $\psi(f)=f \theta^{-1}$. Clearly $\psi(f)=f \theta^{-1} \neq h \theta^{-1}=\psi(h)$ for any distinct good $\gamma_{R}(D)$-functions $f, h \in \mathscr{D}_{i}$ and hence $\psi$ is an injection. On the other hand, for any $g \in \mathscr{D}_{j}$, it holds that $g \theta \in \mathscr{D}_{i}$ and $\psi(g \theta)=(g \theta) \theta^{-1}=g$. Thus, $\psi$ is a bijection from $\mathscr{D}_{i}$ to $\mathscr{D}_{j}$, and so $\left|\mathscr{D}_{i}\right|=\left|\mathscr{D}_{j}\right|$ for any $i, j \in\{1,2, \ldots, n\}$.

If $f=\left(V_{0}, V_{1}, V_{2}\right)$ is a good $\gamma_{R}(D)$-function with $V_{2}=\left\{v_{i_{1}}, \ldots, v_{i_{r}}\right\}$, then $f \in \mathscr{D}_{i_{1}}, \ldots, f \in$ $\mathscr{D}_{i_{r}}$. This implies that every good $\gamma_{R}(D)$-function appears $r=\left|V_{2}\right|$ times in $\cup_{i=1}^{n} \mathscr{D}_{i}$. Thus, the number of good $\gamma_{R}(D)$-functions is equal to $\frac{n\left|\mathscr{D}_{1}\right|}{r}$. Since an $\operatorname{arc}\left(v_{i}, v_{j}\right)$ only supports those good $\gamma_{R}(D)$-functions in $\mathscr{D}_{i}$, we must have $s(D) \geq \frac{n}{r}$ where $s(D)$ is the minimum size of a set of arcs such that each $\gamma_{R}(D)$-function is supported by at least one such arc. Hence $b_{R}(D) \geq$ $s(D) \geq\left\lceil\frac{n}{r}\right\rceil \geq\left\lceil\frac{2 n}{\gamma_{R}(D)}\right\rceil$ and the proof is complete.

\section{Exact values of $b_{R}(D)$}

In this section we determine the exact value of the Roman bondage number of some classes of digraphs.

Theorem 12. If $D$ is a digraph of order $n \geq 3$ with exactly $k \geq 1$ vertices of outdegree $n-1$, then $b_{R}(D)=k$.

Proof. Since $k \geq 1$, we note that $\gamma_{R}(D)=2$ by Proposition A (1). Let $A^{\prime} \subseteq A(D)$ be an arbitrary subset of arcs such that $\left|A^{\prime}\right|<k$, and let $D^{\prime}=D-A^{\prime}$. Clearly, there exists a vertex $w$ in $D^{\prime}$ such that $\operatorname{deg}_{D}^{+}(w)=\operatorname{deg}_{D^{\prime}}^{+}(w)=n-1$ and thus $\gamma_{R}(D)=\gamma_{R}\left(D^{\prime}\right)=2$. This shows that $b_{R}(D) \geq k$.

Now let $v_{1}, v_{2}, \ldots, v_{k}$ be the vertices of outdegree $n-1$ in $D$, and let $a_{i}$ be an $\operatorname{arc}$ of $D$ with tail $v_{i}$ for $1 \leq i \leq k$. Since $D$ has exactly $k$ vertices of outdegree $n-1$, we observe that the maximum outdegree of the digraph $F=D-\left\{a_{1}, a_{2}, \ldots, a_{k}\right\}$ is $n-2$. The hypothesis $n \geq 3$ leads to $\gamma_{R}(F)=3$ by Proposition A (2), and so we see that $b_{R}(D) \leq k$. Altogether, we have $b_{R}(D)=k$, and the proof is complete.

The proof of next result is similar to the proof of Theorem 12 and therefore omitted. 
Theorem 13. If $D$ is a digraph of order $n \geq 3$ with exactly $k \geq 1$ vertices of outdegree $n-1$, then $b(D)=k$.

Corollary 14. If $n \geq 3$, then $b_{R}\left(K_{n}^{*}\right)=b\left(K_{n}^{*}\right)=n$, where $K_{n}^{*}$ is the complete digraph of order $n$.

Corollary 14 reveals that the bounds of Proposition 10 and Theorem 11 are tight.

Proposition 15. Let $K_{n_{1}, n_{2}, \ldots, n_{p}}^{*}$ be the complete $p$-partite digraph such that $p \geq 2 \geq n_{1}, n_{1} \leq$ $n_{2} \leq \cdots \leq n_{p}$ and $n=\sum_{i=1}^{p} n_{i} \geq 3$. Then

$$
b_{R}\left(K_{n_{1}, n_{2}, \ldots, n_{p}}^{*}\right)= \begin{cases}i, & n_{i}=1<n_{i+1} \\ 2 i, & n_{i}=2<n_{i+1}\end{cases}
$$

Proof. Let $V_{1}, V_{2}, \ldots, V_{p}$ be the partite sets of the complete $p$-partite digraph $D=K_{n_{1}, n_{2}, \ldots, n_{p}}^{*}$ with $\left|V_{i}\right|=n_{i}$.

If $n_{1}=1$, then $\gamma_{R}(D)=2$. Let $i$ be the greatest index with $n_{i}=1$. Let $A^{\prime} \subset A(D)$ be a subset of arcs of $D$ that contains one outgoing arc for each vertex $v \in V_{j}$ for $j \leq i$. Then $\gamma_{R}\left(D-A^{\prime}\right) \geq 3$ by Proposition A and thus, $b_{R}(D) \leq i$. On the other hand, if $A^{\prime} \subset A(D)$ is a subset of arcs of $D$ with $\left|A^{\prime}\right| \leq i-1$, then $D-A^{\prime}$ contains a vertex that dominates all other vertices of $D-A^{\prime}$ and thus, $\gamma_{R}\left(D-A^{\prime}\right)=2$. It follows that $b_{R}(D)=i$.

If $n_{1}=2$, then $\gamma_{R}(D)=3$. Let $i$ be the greatest index with $n_{i}=2$. Every $\gamma_{R}(D)$-function $f=\left(V_{0}^{f}, V_{1}^{f}, V_{2}^{f}\right)$ has the property that $V_{1}^{f} \cup V_{2}^{f}=V_{j}$ for an index $j \leq i$. Let $A^{\prime} \subset A(D)$ be a subset of arcs of $D$ that contains one outgoing arc for each vertex $v \in V_{j}$ for $j \leq i$. Then $\gamma_{R}\left(D-A^{\prime}\right) \geq 4$ by Proposition A and hence $b_{R}(D) \leq 2 i$. On the other hand, if $A^{\prime} \subset A(D)$ is a subset of arcs of $D$ with $\left|A^{\prime}\right| \leq 2 i-1$, then $D-A^{\prime}$ contains a set $V_{j}=\{v, w\}$ with $j \leq i$ such that $v$ dominates all vertices of $V(D)-V_{j}$ and thus, $\gamma_{R}\left(D-A^{\prime}\right)=3$. It follows that $b_{R}(D)=2 i$.

Theorem 16. Let $K_{m, n}^{*}$ be the complete bipartite digraph such that $n \geq m \geq 2$ and $m+n \geq 5$. Then

$$
b_{R}\left(K_{m, n}^{*}\right)= \begin{cases}2 & \text { if } m=2 \\ m+2 & \text { if } m \geq 3 .\end{cases}
$$

Proof. The result is immediate for $m=2$ by Proposition 15. Let $m \geq 3$ and let $X=\left\{x_{1}, \ldots, x_{m}\right\}$ and $Y=\left\{y_{1}, \ldots, y_{n}\right\}$ be the partite sets of the complete bipartite digraph $D=K_{m, n}^{*}$. By Proposition $\mathrm{A}$, we have $\gamma_{R}(D)=4$. It is easy to see that $\gamma_{R}\left(D-A^{\prime}\right) \geq 5$ where $A^{\prime}=\left\{\left(x_{i}, y_{1}\right) \mid 1 \leq i \leq\right.$ $m\} \cup\left\{\left(y_{1}, x_{1}\right),\left(y_{1}, x_{2}\right)\right\}$. This yields $b_{R}(D) \leq m+2$.

Now, we show that $b_{R}(D) \geq m+2$. Let $A^{\prime} \subset A(D)$ be a subset of $\operatorname{arcs}$ of $D$ such that $\left|A^{\prime}\right|=$ $m+1$ and let $D^{\prime}=D-A^{\prime}$. Then $D^{\prime}$ has at least $n-1$ vertices whose out-degrees are equal in $D$ and $D^{\prime}$. Let $Z=\left\{v \in V(D) \mid d_{D}^{+}(\nu)=d_{D^{\prime}}^{+}(\nu)\right\}$. If $Z \cap X \neq \varnothing$ and $Z \cap Y \neq \varnothing$, then obviously 
$\gamma_{R}\left(D^{\prime}\right)=4$. Henceforth, we assume that $Z \cap X=\varnothing$ or $Z \cap Y=\varnothing$. Assume that $Z \cap X=\varnothing$ (the case $Z \cap Y=\varnothing$ is similar). Then $Z \subseteq Y$ and $A^{\prime}$ contains one outgoing arc for each vertex $x_{i} \in X$. Since $\left|A^{\prime}\right|=m+1<2 m$, we deduce that $A^{\prime}$ contains exactly one outgoing arc for some $x_{i} \in X$. Assume, without loss of generality, that $i=1$ and $\left(x_{1}, y_{1}\right) \in A^{\prime}$. If $Z=Y$, then $f=\left(V\left(D^{\prime}\right)-\right.$ $\left.\left\{x_{1}, y_{1}\right\}, \varnothing,\left\{x_{1}, y_{1}\right\}\right)$ is a Roman dominating function of $D^{\prime}$ of weight 4 and hence $\gamma_{R}\left(D^{\prime}\right)=4$. Let $Z \varsubsetneqq Y$. Then we may assume that $Z=\left\{y_{1}, \ldots, y_{n-1}\right\}$. Thus $A^{\prime}$ contains one outgoing arc from $y_{n}$, say $\left(y_{n}, x_{m}\right)$. Since $\left|A^{\prime}\right|=m+1$, we conclude that $A^{\prime}$ contains exactly one outgoing arc for each vertex $x_{i} \in X$ and one outgoing arc from $y_{n}$. If $\left(x_{i}, y_{j}\right) \in A^{\prime}$ for some $1 \leq i \leq m$ and some $j<n$, then $f=\left(V\left(D^{\prime}\right)-\left\{x_{i}, y_{j}\right\}, \varnothing,\left\{x_{i}, y_{j}\right\}\right)$ is a Roman dominating function of $D^{\prime}$ of weight 4 and hence $\gamma_{R}\left(D^{\prime}\right)=4$. Thus, we assume that $\left(x_{i}, y_{n}\right) \in A^{\prime}$ for each $1 \leq i \leq m$. But then $\gamma_{R}\left(D^{\prime}\right)=4$ by Proposition A. Thus $b_{R}(D) \geq m+2$ and the proof is complete.

The $k$-th power $D^{k}$ of a directed graph $D$ is defined to be the directed graph on the vertices of $D$ with an arc from $a$ to $b$ in $D^{k}$ if and only if one can get from $a$ to $b$ in $D$ with at most $k$ steps. Figure 1 illustrates the 2 -th power of the directed cycle $C_{9}$.

Theorem 17. For $k \geq 2$, let $C_{n}^{k}$ be the $k$-th power of the directed cycle of length $n \geq 2 k+1$. If $n$ is a multiple of $k+1$, then $b_{R}\left(C_{n}^{k}\right)=k+1$.

Proof. Let $D=C_{n}^{k}$, where $k \geq 2$ and $n$ is a multiple of $k+1$. Let $V=V(D)=\left\{v_{1}, v_{2}, \ldots, v_{n}\right\}$ and $A=A(D)=\left\{v_{i} v_{i+j}: j=1,2, \ldots, k\right\}$ where the subscript $i+j$ is taken modulo $n$. Note that $\gamma_{R}(D)=2 n /(k+1)$ and every $\gamma_{R}(D)$-function $f=\left(V_{0}^{f}, V_{1}^{f}, V_{2}^{f}\right)$ has the property that $V_{1}^{f}=\varnothing$ and $V_{2}^{f}=\left\{v_{i+j(k+1)}: j=0,1, \ldots, n /(k+1)-1\right\}$ for an index $1 \leq i \leq k+1$. Hence, the number of $\gamma_{R}(D)$-functions is $k+1$.

For $i \neq j$, it is easy to see that the function $g: V(D) \rightarrow V(D)$ by $g\left(v_{k}\right)=v_{k+(j-i)}$ for $k=$ $1, \ldots, n$, is an automorphism of $D$ with $g\left(v_{i}\right)=v_{j}$. Therefore, $D$ is a vertex-transitive digraph. It follows from Theorem 11 that $b_{R}(D) \geq k+1$. Now we shall show that $b_{R}(D) \leq k+1$. Let $A^{\prime}=$ $\left\{\left(v_{i}, v_{i+k}\right): i=1,2, \ldots, k+1\right\}$ and $D^{\prime}=D-A^{\prime}$. If $g$ is an arbitrary $\gamma_{R}\left(D^{\prime}\right)$-function, then clearly $V_{1}^{g} \neq \varnothing$ and so $g$ is not a $\gamma_{R}(D)$-function because every $\gamma_{R}(D)$-function $f$ has the property that $V_{1}^{f}=\varnothing$. Therefore, $\gamma_{R}\left(D^{\prime}\right)>\gamma_{R}(D)$ implying that $b_{R}(D) \leq k+1$.

Altogether, we have $b_{R}(D)=k+1$ and the proof is complete.

Using Proposition D and an argument similar to that described in the proof of Theorem 17 , we get the next result.

Theorem 18. For $k \geq 2$, let $C_{n}^{k}$ be the $k$-th power of the directed cycle of length $n \geq 2 k+1$. If $n$ is a multiple of $k+1$, then $\gamma(D)=n /(k+1)$ and $b\left(C_{n}^{k}\right)=k+1$.

Theorems 17 and 18 reveal that the bounds of Propositions 10 and Theorem 11 are tight. 


\section{Acknowledgements}

The authors would like to thank anonymous referees for their remarks and suggestions that helped improve the manuscripts.

\section{References}

[1] J. Amjadi, N. Mohammadi, S. M. Sheikholeslami and L. Volkmann, The k-rainbow bondage number of a digraph, Discuss. Math. Graph Theory, 35 (2015), 261-270.

[2] A. Bahremandpour, F.-T. Hu, S. M. Sheikholeslami and J.-M. Xu, On the Roman bondage number of a graph, Discrete Math. Algorithms Appl. 5 (2013), 1350001 (15 pages)

[3] K. Carlson and M. Develin, On the bondage number of planar and directed graphs, Discrete Math., 306 (2006), 820-826.

[4] E.W. Chambers, B. Kinnersley, N. Prince and D. B. West, Extremal problems for Roman domination, SIAM J. Discrete Math., 23 (2009), 1575-1586.

[5] E. J. Cockayne, P. M. Dreyer Jr., S. M. Hedetniemi, and S.T. Hedetniemi, On Roman domination in graphs, Discrete Math., 278 (2004), 11-22.

[6] N. Dehgardi, D. Meierling, S. M. Sheikholeslami and L. Volkmann, Roman reinforcement numbers of digraphs, J. Combin. Math. Combin. Comput., 89 (2014) 53-64.

[7] N. Dehgardi, S. M. Sheikholeslami and L. Volkmann, On the Roman k-bondage number of a graph, AKCE Int. J. Graphs Comb., 8 (2011), 169-180.

[8] O. Favaron, H. Karami and S. M. Sheikholeslami, On the Roman domination number in graphs, Discrete Math., 309 (2009), 3447-3451.

[9] T. W. Haynes, S. T. Hedetniemi and P. J. Slater, Fundamentals of Domination in Graphs, Marcel Dekker, Inc., New York, 1998.

[10] J. Huang and J.-M.Xu, The bondage numbers of extended de Bruijn and Kautz digraphs, Comput. Math. Appl., 51 (2006), 1137-1147.

[11] J. Huang and J.-M. Xu,The bondage numbers and efficient dominations of vertex-transitive graphs, Discrete Math., 308 (2008), 571-582.

[12] J. Huang and J.-M. Xu, The total domination and total bondage numbers of extended de Bruijn and Kautz digraphs, Comput. Math. Appl., 53 (2007), 1206-1213.

[13] N. J. Rad and L. Volkmann, Roman bondage in graphs, Discuss. Math. Graph Theory, 31 (2011), 763-773.

[14] N. J. Rad and L. Volkmann, On the Roman bondage number of planar graphs, Graphs Combin., 27 (2011), 531-538.

[15] C.S. Revelle and K.E. Rosing, Defendens imperium romanum: a classical problem in military strategy, Amer. Math. Monthly, 107 (2000), 585-594.

[16] E. Shan and L. Kang, Bondage number in oriented graphs, Ars Combin., 84 (2007), 319-331.

[17] S. M. Sheikholeslami and L. Volkmann, The Roman domination number of a digraph, Acta Univ. Apulensis Math. Inform., 27 (2011), 77-86.

[18] I. Stewart, Defend the Roman Empire, Sci. Amer., 281 (1999), 136-139.

[19] J.-M. Xu, On bondage numbers of graphs: a survey with some comments, Int. J. Combin. Volume 2013, Article ID 595210, 34 pages.

[20] D. B. West, Introduction to Graph Theory, Prentice-Hall, Inc, 2000.

Department of Mathematics and Computer Science, Sirjan University of Technology, Sirjan, I.R. Iran.

E-mail: ndehgardi@gmail.com

Lehrstuhl II für Mathematik, RWTH Aachen University, 52056 Aachen, Germany. 
E-mail: meierling@math2.rwth-aachen.de

Department of Mathematics, Azarbaijan Shahid Madani University, Tabriz, I.R. Iran.

E-mail: s.m.sheikholeslami@azaruniv.edu

Lehrstuhl II für Mathematik, RWTH Aachen University, 52056 Aachen, Germany.

E-mail: volkm@math2.rwth-aachen.de 